\title{
Single-Stage Peninsula-Shaped Lateral Tongue Flap Reconstruction for Buccal Defects in Two Patients: Revisiting a Simple and Safe Reconstructive Option
}

\author{
Yu Taek Kong ${ }^{1 *}$ (D), Soo Yeon Lim²,3* (D), Junekyu Kim¹ (D), Hyun Woo Shin ${ }^{1}$ (D), Kyu Nam Kim ${ }^{1}$ (D) \\ ${ }^{1}$ Department of Plastic and Reconstructive Surgery, Kangbuk Samsung Hospital, Sungkyunkwan University School of Medicine, Seoul; ${ }^{2}$ Department of Plastic \\ and Reconstructive Surgery, Konyang University Hospital, Daejeon; ${ }^{3}$ Myunggok Medical Research Center, Konyang University College of Medicine, Daejeon, \\ Korea
}

\begin{abstract}
Intraoral defects are increasingly reconstructed using free flaps. However, certain situations may render free flap reconstruction difficult. This study presents two cases with underlying comorbidities that underwent reconstruction of buccal defects involving the retromolar trigone (RMT), using a single-stage peninsula-shaped lateral tongue flap (pLTF). A 58-year-old woman and a 75-year-old man were diagnosed with left buccal squamous cell carcinoma and right buccal adenoid cystic carcinoma, respectively. The buccal defects extending to the RMT area were detected and covered with an ipsilateral posteriorly based pLTF after wide excision of the lesion. All flaps survived without any postoperative complications. Moreover, no tumor recurrences or functional problems were observed during the final follow-up. A single-stage pLTF is believed to represent a good alternative to free flaps in buccal and RMT defects.
\end{abstract}

Keywords: Tongue; Flap; Pedicled flap; Buccal mucosa; Reconstruction

\section{Introduction}

Intraoral soft tissue defects develop because of various conditions, such as tumor resection, congenital anomalies, and traumatic injuries [1]. Choosing an appropriate reconstructive option is vital in restoring form and function and improving the patients' quality of life [1-5]. The goal of intraoral defect reconstruction, similar to other reconstructive surgeries, is to provide reliable and durable coverage of the defect with minimal donor site morbidity, both functionally and morphologically [6,7]. Herein we present two cases with underlying comorbidities that underwent reconstruction of buccal defects involving the retromolar trigone (RMT), using single-stage posteriorly based peninsula-shaped lateral tongue flaps (pLTFs). This study aims to revisit the tongue flap technique as a primary reconstructive modality, demonstrate its utility, and expand its versatility in intraoral defect coverage. This study was approved by the Institutional Review Board of Konyang University Hospital (approval number: 2020-11-019). All research procedures were performed following the ethical guidelines of the 1975 Declaration of Helsinki. Written informed consent was obtained from all patients.
Case Report

Received: December 30, 2020

Revised: March 16, 2021

Accepted: March 17, 2021

Corresponding author:

Kyu Nam Kim, M.D., Ph.D.

Department of Plastic and Reconstructive Surgery, Kangbuk Samsung Hospital,

Sungkyunkwan University School of Medicine,

29 Saemunan-ro, Jongno-gu, Seoul 03181,

Korea

Tel: +82-2-2001-2182

Fax: +82-2-2001-2117

E-mail: manabear77@naver.com

*These authors contributed equally to this work.

This article was presented as an e-poster at PRS KOREA 2019 on November 8-10, 2019.

This is an Open Access article distributed under the terms of the Creative Commons Attribution Non-Commercial .//creativecommons. org/licenses/by-nc/4.0/) which permits unrestricted non-commercial use, distribution, and reproduction in any medium, provided the original work is properly cited.

(C) 2021 Korean Wound Management Society 


\section{Cases}

\section{Case presentation}

Case 1

A 58-year-old woman presented with a whitish mass-like lesion in the left upper buccal mucosa (Fig. 1). She had multiple comorbidities, including hypertension and diabetes mellitus. Moreover, she was diagnosed with squamous cell carcinoma involving the left buccinator muscle, adjacent buccal fat pad, and orifice of the Stensen's duct with no evidence of distant metastasis on preoperative staging workup.

\section{Case 2}

A 75-year-old man presented with a protruding hard masslike lesion in the right cheek area (Fig. 2). He had multiple comorbidities, including hypertension, diabetes mellitus, and asthma. Consequently, he was diagnosed with adenoid cystic carcinoma involving the right buccinator muscle and extending to the platysma, with no evidence of distant metastasis on preoperative staging workup.

\section{Surgical procedure}

Each patient underwent wide excision of the lesions and ipsi- lateral selective neck dissection (levels I, II, and III). All resection margins were negative, as was confirmed by the senior pathologist of this study. The final defect size for case 1 was $6 \times 4 \mathrm{~cm}$ and involved the left upper buccal mucosa extending to the RMT area, while in case 2 it was $5 \times 4 \mathrm{~cm}$, involving the right lower buccal mucosa extended to the RMT area. A posteriorly based pLTF was applied in both cases. For ease of manipulation, the midline groove of the tongue was marked by placing a tagging suture made of 3-0 black silk at the apex. The lateral tongue flap (LTF) was designed on the side ipsilateral to the defect. The maximal length of the flap was made equal to that of the mobile tongue ( $>7 \mathrm{~cm}$ in both cases), whereas the width of the flap was designed to be slightly larger than that of the defect. Furthermore, the flap was created by a full-thickness incision extending from the anterior to the posterior direction, using electrocautery. The flap included a cuff of the intrinsic muscle attached to the mucosal layer, and to prevent vasculature injury, its elevation did not extend beyond the circumvallate papilla. Moreover, a minimal mucosal bridge was maintained at the pivot point to ensure additional vascular channels to the flap, to protect the pedicle, and to allow for safe flap handling at the time of transfer. The donor site was primarily closed without any tension. Thereafter, the mucosal
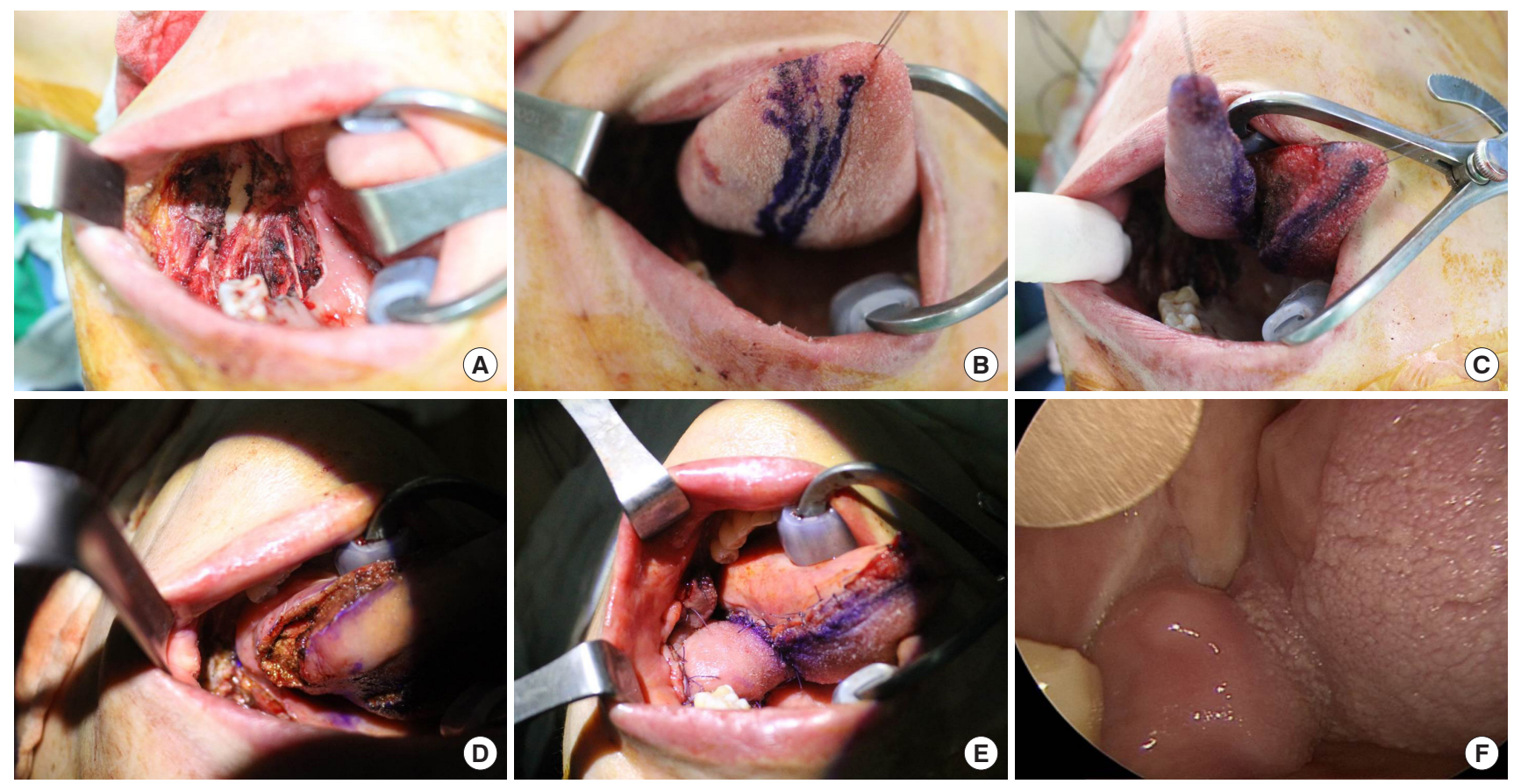

Fig. 1. Clinical photographs of case 1. (A) The final defect $(6 \times 4 \mathrm{~cm})$ in the left buccal area involving the retromolar trigone. (B) The design of the posteriorly based lateral tongue flap. (C) Flap elevation. (D) Maintenance of the minimal mucosal bridge at the pivot point, and removal of the mucosa buried below the flap. (E) Immediate postoperative photograph. (F) Postoperative photograph after the 12 months follow-up. 
layer between the flap pivot point and the defect was incised and opened up to allow the flap rotation to reach the defect. The flap was eventually sutured onto the defect, creating a peninsula-like shape. Fig. 3 presents the stepwise schematic illustration of this approach.

\section{Results and postoperative adjuvant treatment}

The flap elevation time was 35 and 30 minutes in cases 1 and 2, respectively. Consequently, all flaps survived without any complications such as flap loss, wound dehiscence, or fistula formation. The pathologic TNM stages were pT1N0M0 and pT-
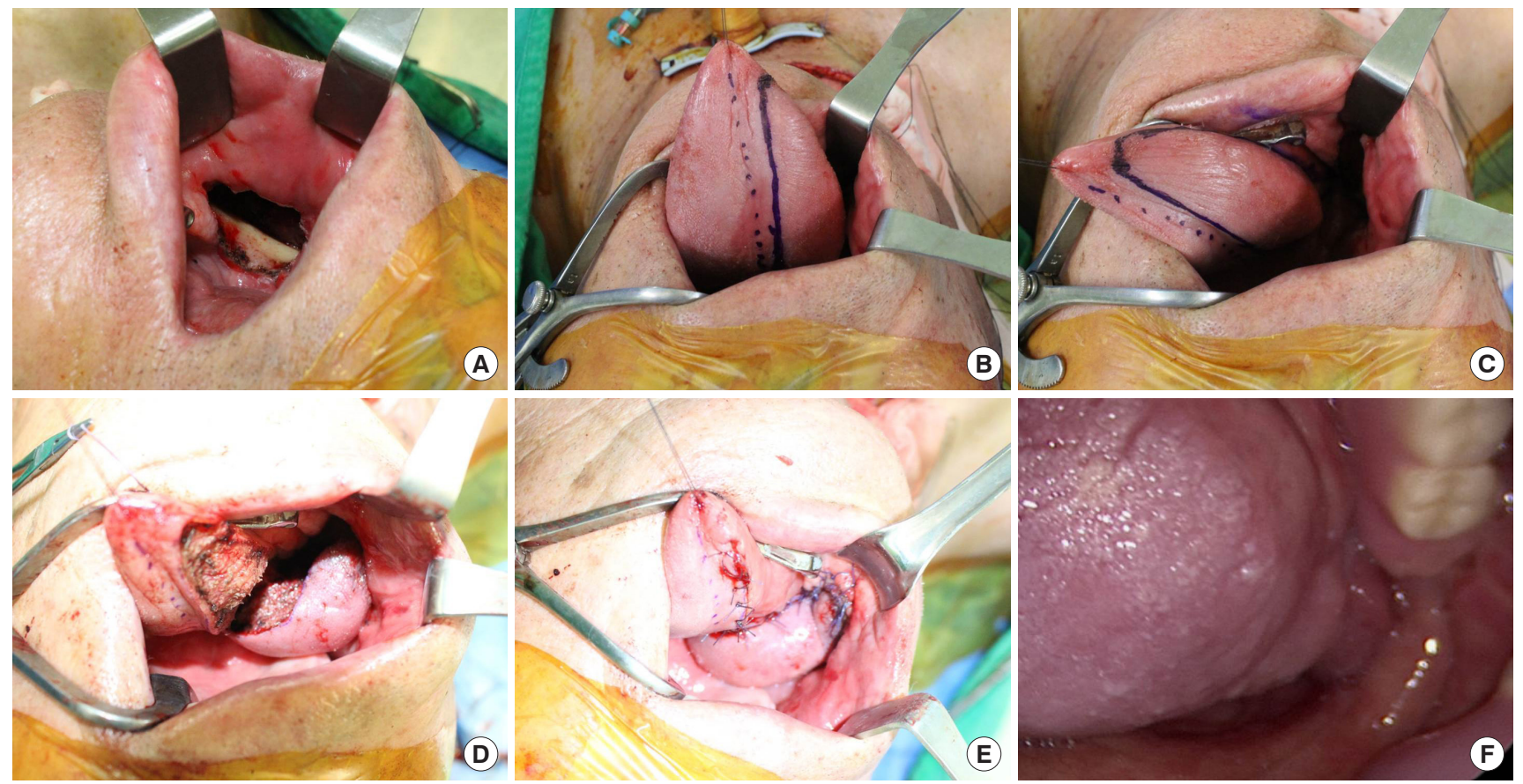

Fig. 2. Clinical photographs of case 2. (A) The final defect $(5 \times 4 \mathrm{~cm})$ in the right buccal area involving the retromolar trigone. (B, C) Design of the posteriorly based lateral tongue flap. (D) Elevation of the flap, maintenance of the minimal mucosal bridge at the pivot point, and removal of the mucosa buried below the flap. (E) Immediate postoperative photograph. (F) Postoperative photograph after 11 months of follow-up.
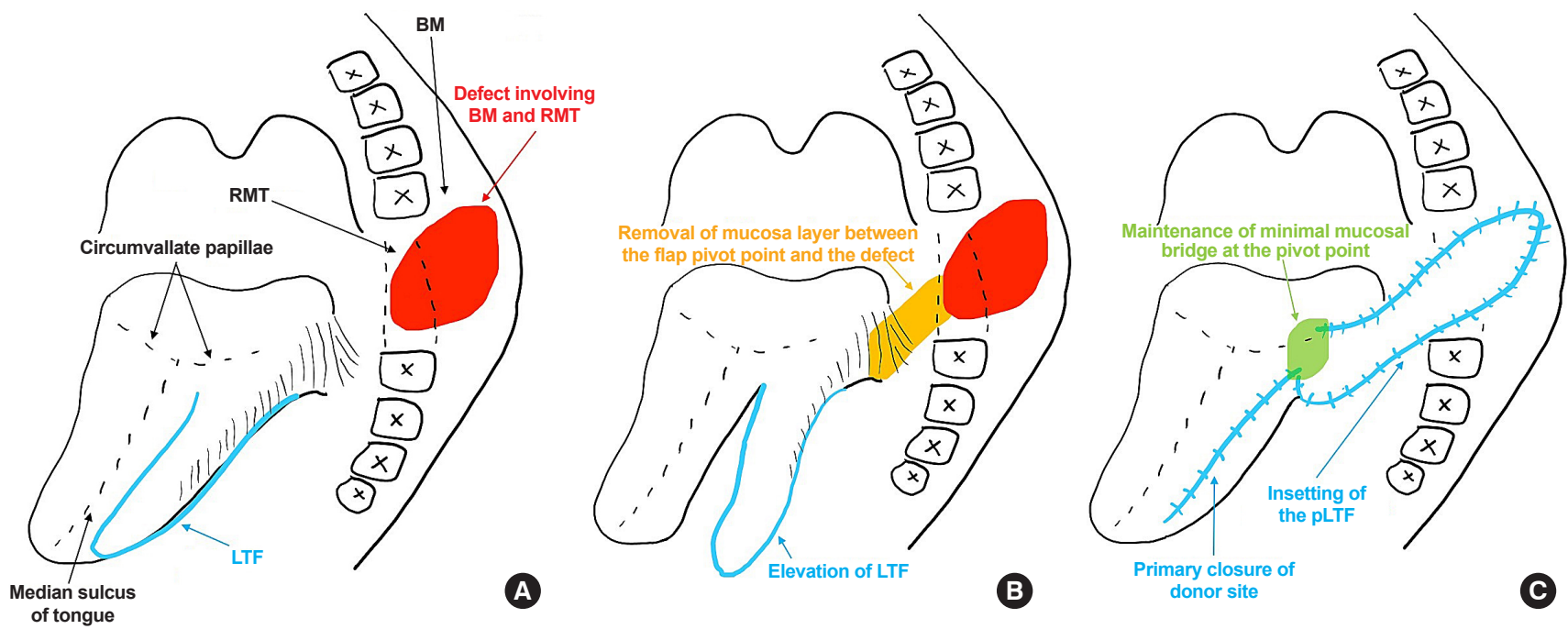

Fig. 3. Schematic illustration of the single-stage pLTF. (A) Design of the posteriorly based LTF. (B) Elevation of the LTF and removal of the mucosa layer between the flap pivot point and the defect to allow flap rotation to reach the defect. (C) Insetting of the pLTF and primary closure of the donor site. LTF, lateral tongue flap; pLTF, peninsula-shaped LTF; RMT, retromolar trigone; BM, buccal mucosa. 
3N0M0, and postoperative 1-month radiation therapy with 54 Gy in 30 fractions and 66 Gy in 33 fractions was administered to prevent local tumor recurrence and increase disease-free survival rate, respectively. No tumor recurrences or problems with tongue movement or phonation were observed in the 12 and 11 months follow-up of each respective patient.

\section{Discussion}

This study presented the experience of single-stage reconstruction of buccal defects involving the RMT area using a posteriorly based pLTF in two cases. Good outcomes were obtained in both cases. Free flap reconstruction, which has superior functional and aesthetical outcomes to other methods, is increasingly applied due to recent advancements in microsurgical concepts and skills [5]. Nevertheless, the use of free flaps has its limitations, mainly in terms of cost, need for microsurgery expertise and postoperative care, and underlying comorbidities that prohibit lengthy operations $[5,8]$. Thus, the local flap technique can be a good alternative in such circumstances, despite its relatively limited application in the intraoral region [2,5]. Local flaps are especially useful in intraoral defects which are neither small enough to be closed primarily nor large enough to require free flaps [5].

Since their introduction by Lexer in 1909, LTFs have conferred distinct advantages, including reliable flap perfusion owing to their rich vasculature, close similarity to adjacent tissues, and technical simplicity (i.e., no need for microvascular surgery) [3-6,9]. However, the technique has not been widely adopted as a primary reconstructive option despite being the first tongue flap to be devised. The underuse of LTFs may result from the fear of interfering with tongue functions (e.g., swallowing, phonation, and taste) upon tongue tissue removal $[2,4,5]$. However, the outcomes of several studies demonstrate that such fear is unwarranted $[2,4,5]$. No significant functional deficits of the tongue were observed in either patient in the present study, as well as from previous studies.

The short flap harvest time is another advantage of LTFs in addition to their technical simplicity [4]. A previous study reported an average harvest time of 25 minutes [4], which was shorter than in the cases of this study (32.5 minutes). Shorter operative times result in fewer morbidities and complications in patients with underlying comorbidities [8]. Therefore, the LTF approach can be a good option for such patients. Furthermore, the pLTF, which maintained minimal mucosal bridge at the pivot point, provides vascular support and structural stabi- lization of the flap and helps shorten the operation by removing the need for incising and dissecting around the areas of the mucosal bridge [10]. In addition, a single-stage operation is crucial for managing patients with comorbidities, as in the current cases. The key aspect when performing single-stage reconstructions without a second operation to divide the pedicle in the posteriorly based pLTF is that the mucosa interposed between the donor site and the defect should be raised or removed [9]. In conclusion, this study presents successful cases of single-stage pLTF reconstruction following tumor resection in the buccal area involving the RMT region. It is believed that no reports of buccal and RMT defect coverage using the single-stage tongue flap exist, and the present study may be the first case report in Korea. This study proposes that the posteriorly based pLTF technique can be a good reconstructive option for intraoral defects because of its technical ease, safety, and favorable outcomes in selected cases with relatively small-sized defects, even if the patients have multiple underlying comorbidities.

\section{Conflict of interest}

No potential conflict of interest relevant to this article was reported.

\section{ORCID iDs}

Yu Taek Kong

Soo Yeon Lim

https://orcid.org/0000-0001-6267-4463

Junekyu Kim

Hyun Woo Shin

https://orcid.org/0000-0002-2954-2191

Kyu Nam Kim

https://orcid.org/0000-0002-8904-5605

https://orcid.org/0000-0003-4396-3395

https://orcid.org/0000-0001-9998-8722

\section{References}

1. Comini LV, Spinelli G, Mannelli G. Algorithm for the treatment of oral and peri-oral defects through local flaps. J Craniomaxillofac Surg 2018;46:2127-37.

2. Ceran C, Demirseren ME, Sarici M, et al. Tongue flap as a reconstructive option in intraoral defects. J Craniofac Surg 2013;24:972-4.

3. Fujiwara K, Koyama S, Fukuhara T, et al. Primary Closure with posteriorly based lateral tongue flap reconstruction after transoral videolaryngoscopic surgery for tonsil cancer. Ear Nose Throat J 2020 Aug 19 [Epub]. https://doi. org/10.1177/0145561320949692. 
4. Kumar V, Mukharjee S, Akhtar N, et al. Tongue flap reconstruction in carcinoma of oral cavity: an institutional experience. J Maxillofac Oral Surg 2019;18:428-31.

5. Deshmukh A, Kannan S, Thakkar P, et al. Tongue flap revisited. J Cancer Res Ther 2013;9:215-8.

6. Strauss RA, Kain NJ. Tongue flaps. Oral Maxillofac Surg Clin North Am 2014;26:313-25.

7. Lee HG, Lim SY, Yoon CS, et al. Circumferential penile defect reconstruction with pull-up double-opposing keystone-designed perforator island flaps: a case report. Medicine (Baltimore) 2020;99:e18762.
8. Yoon CS, Kim SI, Kim H, et al. Keystone-designed perforator island flaps for the coverage of traumatic pretibial defects in patients with comorbidities. Int J Low Extrem Wounds 2017;16:302-9.

9. Cordova A, Toia F, D’Arpa S, et al. A new mucosal propeller flap (deep lingual artery axial propeller): the renaissance of lingual flaps. Plast Reconstr Surg 2015;135:584e-594e.

10. Yoon CS, Kim CG, Kim H, et al. Retrospective study of freestyle perforator- based peninsular flaps: a simple, fast, and safe technique for pressure sore reconstruction. Medicine (Baltimore) 2018;97:e0168. 